\title{
Insights into the Management of Papillary Microcarcinoma of the Thyroid
}

\author{
Akira Miyauchi, Yasuhiro Ito, and Hitomi Oda
}

Background: Rapid increases in the incidence of thyroid carcinoma with stable mortality rates from thyroid carcinoma have been reported from many countries, and these increases are thought to be due mostly to the increased detection of small papillary thyroid carcinomas (PTCs), including papillary microcarcinomas (PMCs; i.e., PTCs $\leq 10 \mathrm{~mm}$ ). Some researchers have suggested that small PTCs have been overdiagnosed and overtreated. In Japan, the active surveillance of patients with low-risk PMCs was initiated by Kuma Hospital (1993) and Tokyo's Cancer Institute Hospital (1995) based on the extremely higher incidences of both latent thyroid carcinomas in autopsy studies and small PTCs detected in mass screening studies using ultrasound examinations compared to the prevalence of clinical thyroid carcinomas.

Methods: The above two institutions' data are summarized regarding the active surveillance of low-risk PMCs, and future prospects for their management are discussed.

Results: At 10-year observations in the Kuma Hospital series of 1235 patients, only $8 \%$ and $3.8 \%$ of the PMC patients showed size enlargement by $\geq 3 \mathrm{~mm}$ and the novel appearance of node metastasis, respectively. In contrast to clinical PTC, PMCs are most unlikely to grow in older patients ( $\geq 60$ years). In the Kuma Hospital series, the 974 patients who underwent immediate surgery had significantly higher incidences of unfavorable events than the 1179 patients who chose active surveillance. The total cost of immediate surgery, including the costs for salvage surgery and postoperative care for 10 years, was 4.1 times the total cost of 10-year management by active surveillance. Only $8 \%$ of the 51 PMC patients showed tumor enlargement during pregnancy, and the rescue surgeries after delivery were successful. In the Cancer Institute Hospital series of 230 patients with 300 lesions, only $7 \%$ and $1 \%$ of the patients showed size enlargement and novel node metastasis, respectively, and that institution's analysis also revealed that macroscopic or rim calcification and poor vascularity were correlated with non-progressing disease. In both series, none of the patients who underwent rescue surgery after progression signs were detected showed significant recurrence or died of PTC.

Conclusion: Active surveillance of low-risk PMC can be the first-line management. Interestingly, older patients with low-risk PMCs are the best candidates for active surveillance.

Keywords: papillary microcarcinoma, thyroid, active surveillance, risk classification, surgery, unfavorable events, medical cost

\section{Background}

$\mathbf{R}$ APID INCREASES IN THYROID cancer incidence have been reported in many countries, including the United States, Korea, and Japan (1-4). These increases are due mostly to an increase in the incidence of small papillary thyroid carcinomas (PTCs), whereas the incidences of large PTCs and malignancies of other histological types have remained stable (3). The mortality rate from thyroid cancer has remained fairly stable (1-3). Several research groups have therefore suggested that small PTCs have been overdiagnosed and overtreated (1-3). Papillary thyroid carcinomas that are $\leq 10 \mathrm{~cm}$, which are known as papillary microcarcinomas (PMCs), were demonstrated to account for $\geq 50 \%$ of recent thyroid cancer series (1-3). A major clinical issue has arisen: how best to manage patients with PMCs.

The definition of PMC is a PTC measuring $\leq 10 \mathrm{~mm}$, regardless of the presence/absence of high-risk features such as vocal cord paralysis, clinically apparent lymph node metastasis, and distant metastasis. The term "PMC" thus covers

(C) Akira Miyauchi et al. 2018; Published by Mary Ann Liebert, Inc. This is an Open Access article distributed under the terms of the Creative Commons Attribution License, which permits unrestricted use, distribution, and reproduction in any medium, provided the original work is properly cited. 
a broad range of biological characteristics. Sugitani and $\mathrm{Fu}-$ jimoto reported a striking difference in the prognosis after surgery between patients with symptomatic PMCs and those with asymptomatic PMCs. In that study, the patients with symptomatic PMC with clinically apparent lymph node metastasis and/or vocal cord paralysis group showed a 30\% recurrence rate and a $74.1 \%$ rate of cause-specific survival at 10 years, whereas the asymptomatic PMCs without those features had corresponding rates of $3 \%$ and $100 \%$, respectively (5).

As reported in 2010, patients with PMCs without clinical node or distant metastasis also had excellent prognoses after surgery without postoperative radioactive iodine treatment (6). PMCs with aggressive features should be treated appropriately depending on the extent of disease progression. However, the vast majority of PMCs are asymptomatic tumors lacking aggressive features, detected incidentally by imaging studies or thyroid screening studies. The optimal management of these incidentally detected asymptomatic PMCs is a major clinical issue, and it is the focus of this review.

In autopsy studies of individuals who died of non-thyroid diseases, latent 3-10 mm PMCs were detected in 0.5-5.2\% of subjects (7). With an ultrasound examination and ultrasoundguided fine-needle aspiration biopsy (FNAB), PMCs of this size can be easily detected and diagnosed. Takebe et al. conducted a thyroid cancer mass screening using ultrasound and FNAB on 1048 women at Kagawa Cancer Screening Center's breast cancer screening, and thyroid carcinoma was detected in $3.5 \%$ of the otherwise healthy Japanese women aged $\geq 30$ years (8). Of these thyroid carcinomas, $85 \%$ were $\leq 15 \mathrm{~mm}$. This incidence was curiously almost the same as those of latent thyroid cancer in autopsy studies and $>1000$ times the prevalence of clinical thyroid carcinoma in Japanese women reported at that time. These findings support the concept of PMC overdiagnosis and overtreatment.

\section{Initiation of the Active Surveillance of Low-Risk PMCs}

In the early 1990s, the natural history of PMCs was unknown, and their natural history remains to be further elucidated. The most commonly held idea was that PMCs are at an early stage of clinical cancer that might kill the host if not treated appropriately. However, based on the large differences between the thyroid carcinoma incidences in autopsy studies and the screening study and the prevalence of clinical thyroid carcinoma, Miyauchi hypothesized that most PMCs do not grow or grow very slowly. He speculated that only a small minority of PMCs would grow. Any advanced cancer was of course a small cancer at its beginning. Miyauchi also suspected that a watchful follow-up (i.e., observation) could detect PMCs that grew and that rescue surgery after the detection of PMC progression signs would not be too late to control the carcinoma, and that carrying out surgery for all PMCs would result in more harm than good.

In 1993, Miyauchi proposed an observation without immediate surgery clinical trial for low-risk PMCs at a physicians' meeting at Kuma Hospital. This trial was approved and began that year. The observation without surgery design later came to be called "active surveillance." In 1995, the Cancer Institute Hospital (CIH; Tokyo) also initiated an active-surveillance program for low-risk PMCs, and these two institutions have published promising data using large patient series.

The diagnosis of PMCs was made based on the results of ultrasound-guided FNABs for nodules $\geq 5 \mathrm{~mm}$ with suspicious ultrasound features, and the positive predictive value for diagnosing papillary carcinoma was approximately $98 \%$. Surgery was recommended to patients with high-risk PMCs. For patients with low-risk PMCs, active surveillance or immediate surgery was proposed as alternatives, and the patient was asked to choose one of these options. Patients who chose active surveillance were followed with ultrasound examinations performed six months after the diagnosis and once a year thereafter. Surgery was recommended when tumors increased by $\geq 3 \mathrm{~mm}$ or when novel lymph node metastasis appeared. A few patients were prescribed levothyroxine to maintain their serum thyrotropin (TSH) at low normal or mildly suppressed levels.

When the active surveillance began, the inclusion and exclusion criteria for active surveillance were determined, and these criteria are essentially the same today. There are two types of contraindications-high-risk features and features unsuitable for active surveillance-although it remains unclear whether the latter are biologically aggressive features (Table 1). High-risk features include (i) clinical node positivity (N+; Fig. 1) or clinical distant metastasis positivity (M+; very rare); (ii) signs or symptoms of invasion to the recurrent laryngeal nerve (RLN) or trachea (Fig. 2); (iii) highgrade malignancy such as tall-cell variant on cytology (very rare for PMCs); and (iv) the presence of progression signs (i.e., size enlargement and/or the novel appearance of lymph node metastasis) during active surveillance. PMCs shown by imaging to be attached to the trachea or located on the course of the RLN are also included in the contraindication category

Table 1. Contraindications for the Active Surveillance of PMCs

Type Contraindications

Clinical high-risk features

Features unsuitable for observation, although it is unclear whether they are associated with biological aggressiveness
1. N1 (may present on imaging studies) or M1 (very rare)

2. Signs or symptoms of invasion to the recurrent laryngeal nerve or trachea

3. High-grade malignancy on cytology (very rare)

4. Cases showing progression signs such as size enlargement or a novel appearance of lymph node metastasis during active surveillance

Imaging studies indicate that the tumor may invade the trachea or recurrent laryngeal nerve

PMC, papillary microcarcinoma. 

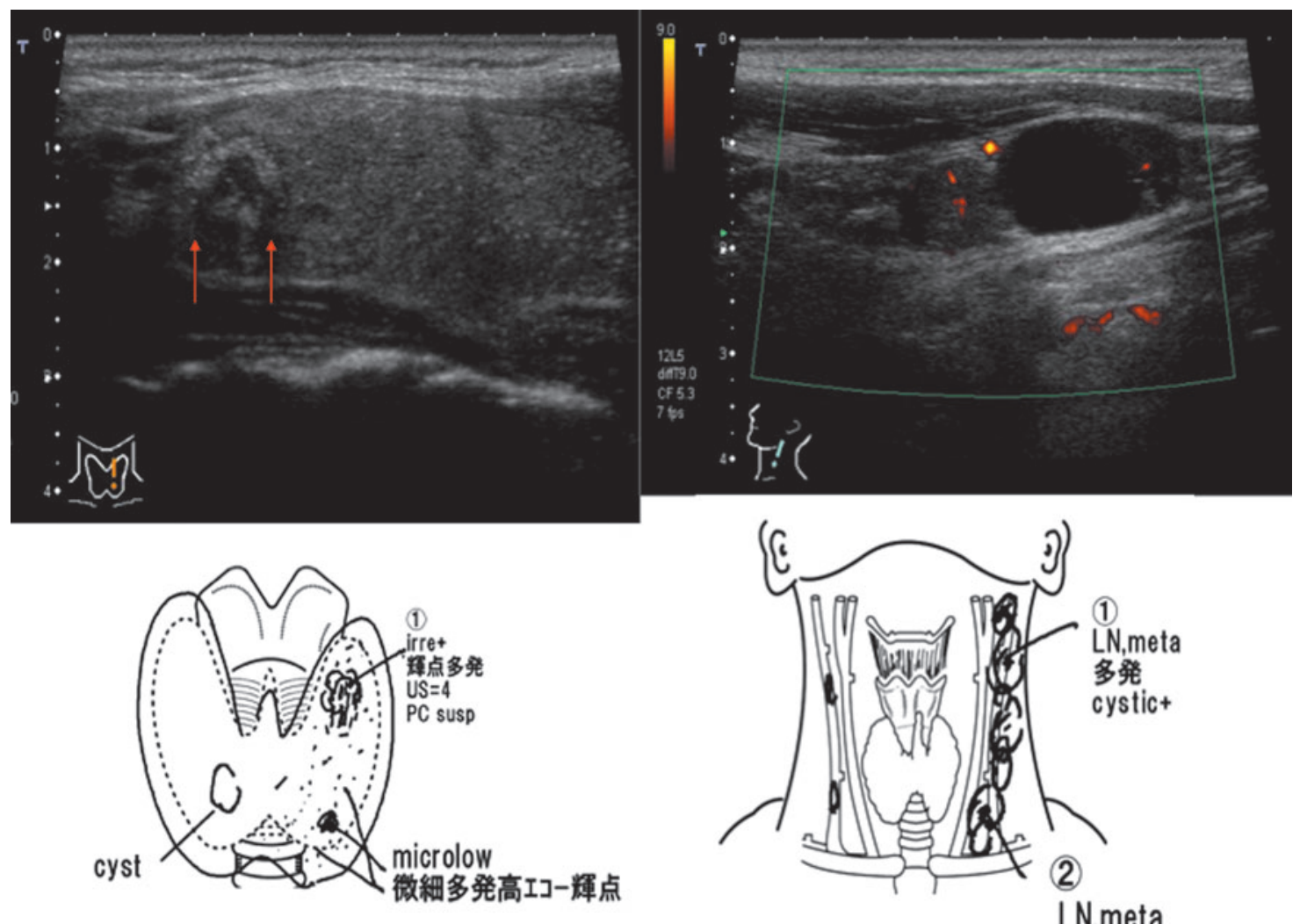

FIG. 1. Papillary microcarcinoma (PMC) with clinically apparent lymph node metastasis (41-year-old woman). Left: a tumor measuring $1 \mathrm{~cm}$ and multiple hyperechoic spots in the left lobe. Right: Multiple metastatic nodules in the left lateral neck compartment. LN, meta, lymph node metastasis; PC, papillary carcinoma; Irre, irregular shape; UC =4, ultrasound classification class 4 (Kuma Hospital Classification); microlow, very small hypoechoic lesion; cystic+, presence of cystic change.

cautiously, although it is unclear whether these tumors are biologically aggressive.

Notably, tumor multiplicity and a family history of differentiated thyroid carcinoma are not included in the contraindications. Tumors in patients with a positive family history tend to be multiple, and multiple PMCs show high incidences of pathological nodal metastasis. However, the data showed that these were not strong prognostic factors. If these elements are included in the indications for surgery, the incidence of total thyroidectomy would become high, thus possibly resulting in higher rates of complications. Therefore, these features were not included in the contraindication category.

\section{Recent Findings Regarding Thyroid Carcinoma Incidence and Mortality}

As noted above, great differences between the incidence and mortality of thyroid carcinoma in various countries have been reported (1-4). In the United States, the thyroid carcinoma incidence increased by 2.4-fold and 2.9-fold between 1973 and 2002 and between 1975 and 2009, respectively (1,2). In Korea, the incidence of thyroid carcinoma increased even more radically, with a 15-fold increase between 1993 and 2011 (3). An increased thyroid carcinoma incidence was also reported in Italy, France, England, Scotland, Australia, and the Nordic countries (9). However, importantly, the thyroid carcinoma
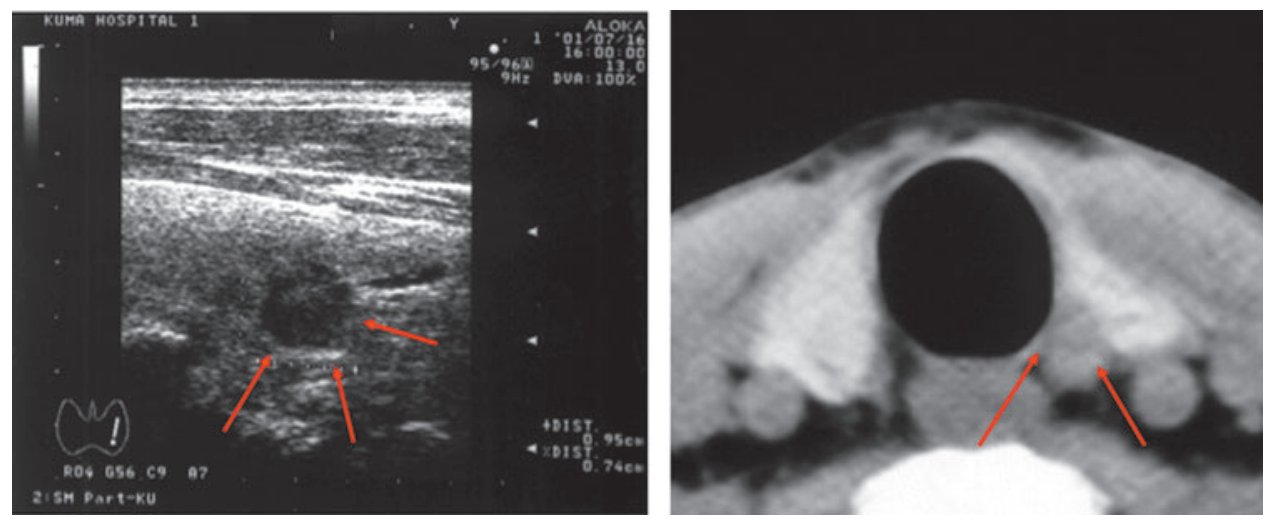

FIG. 2. PMC with left vocal cord paralysis due to carcinoma invasion (74-yearold man). Left: An ultrasonogram showing a hypoechoic tumor (arrows) extending from the dorsal surface of the left thyroid lobe. Right: A computed tomography scan revealing a low-density tumor (arrows) located on the course of the left recurrent laryngeal nerve. 
mortality rate remained stable during the observation period. In the United States, an increased incidence of small papillary carcinomas, including PMC, was also reported $(1,2)$, which was the main reason for the difference between the incidence and mortality rates. Recently, however, one study reporting that incidence-based mortality increased $1.1 \%$ per year during 1994-2013 was published (10), indicating that the change in mortality of thyroid cancer remains debatable.

\section{Active Surveillance of Low-Risk PMC}

Figure 3 illustrates the management flow for patients with low-risk PMCs. The 2015 American Thyroid Association (ATA) management guidelines for adult patients with thyroid nodules and differentiated thyroid cancer do not recommend FNAB for nodules suspected of being low-risk PMC (11). Currently, at Kuma Hospital, PMCs $\geq 5 \mathrm{~mm}$ are diagnosed on cytology, and patients are notified of the diagnosis. There are two reasons for this approach. The size cutoff for performing FNAB for thyroid nodules with suspicious sonographic features in the Japan Association of Breast and Thyroid Sonology guidelines is $5 \mathrm{~mm}$. If an FNAB is not performed, the patient might then consult another physician who might perform an FNAB, and the patients may be told both that Kuma Hospital "missed the diagnosis" of thyroid cancer and that they should undergo surgery immediately. This is an unacceptable scenario for the patients and Kuma Hospital. The second reason that patients are notified of the diagnosis is to encourage them to undergo regular checkups during active surveillance. Without the diagnosis of PMC, this would not be easy. Regular check-ups are considered necessary for patients with suspicious nodules, since some of these cases do show disease progression.

The most important issue is to determine whether a PMC is suitable for active surveillance based on imaging such as ultrasound and computed tomography (CT) scan if necessary. Physicians should carefully check whether a tumor shows the possibility of invading the trachea and/or RLN and whether clinical node metastasis is detected. An FNAB for suspicious nodes, together with a thyroglobulin ( $\mathrm{Tg}$ ) measurement of the FNAB needle washout (12), is useful to confirm nodal metastasis.
After diagnosing a nodule as a PMC suitable for active surveillance, in the past, two management options (active surveillance vs. immediate surgery) were presented rather equally to the patients, and the patients chose which option they wished to pursue. This was because confirmed evidence was lacking, although it was suspected that active surveillance would be better for the patients than immediate surgery. At present, active surveillance is recommended as the first-line management for lowrisk PMCs based on the accumulated evidence.

Patients who choose active surveillance are followed with ultrasound at six months after the diagnosis and at least once a year thereafter. If suspicious nodes are detected, a FNAB and Tg measurement of the FNAB needle washout for these nodes (12) are performed to determine whether the nodes are metastatic or reactive. Rescue surgery is recommended when the tumor size has increased by $\geq 3 \mathrm{~mm}$, but if the patient prefers active surveillance, it can be continued until the tumor size reaches $13 \mathrm{~mm}$. Cases with newly appearing lymph node metastasis are strong candidates for rescue surgery. Without these events, patients are followed with continuing active surveillance.

\section{Data on Active Surveillance of Low-Risk PMC}

In 2003, the first report of active surveillance was published from Kuma Hospital, demonstrating that the size of $>70 \%$ of the PMCs did not change or even decreased compared to that at the initial measurement (13). Kuma Hospital's second report was published in 2010, increasing the number of enrolled patients to 340 (14). It showed that various clinicopathologic features (e.g., sex, family history, and multiplicity) were not related to PMC progression, but PMCs in young patients (i.e., $<45$ years) were likely to progress, although the difference was not significant. The third clinical study, published in 2014 (15), enrolled 1235 patients. Only 8\% of the PMC showed a size enlargement and only $3.8 \%$ were found have a novel appearance of node metastasis after a 10-year observation. Important findings about the relationship between age and PMC progression were also demonstrated.

In clinical PTCs, old age is the most important factor in terms of mortality from thyroid carcinoma (16). Another investigation showed that young age (i.e., <30 years) and old age ( $\geq 60$ years) were independent prognostic factors of
FIG. 3. Flow of the management of patients with low-risk PMC. AS, active surveillance; US, ultrasound.

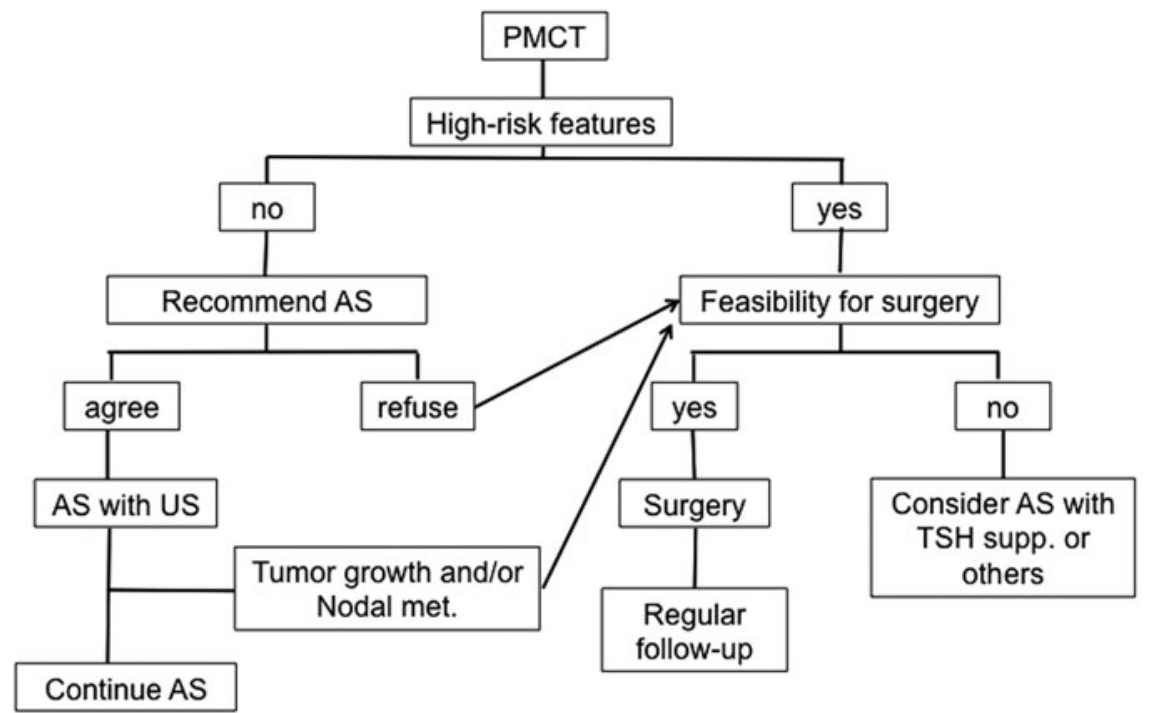


Table 2. Results and Findings of Observation for Low-Risk PMC at Kuma Hospital and the Cancer Institute Hospital

Kuma Hospital

1. Of 1235 patients, $8 \%$ and $3.8 \%$ showed size enlargement and novel node metastasis, respectively, at 10 -year observation (15).

2. The PMCs of young patients are likely to progress, and those of old patients are most unlikely to grow (15).

3. None of the patients who underwent surgery after the detection of progression signs showed significant recurrence or died of PTC (15).

4. Only $8 \%$ of the patients showed PMC progression during pregnancy, and rescue surgery after delivery was successful (29).

5. Immediate surgery and active surveillance managements had similarly excellent oncological outcomes. However, the immediate surgery resulted in significantly higher incidences of unfavorable events than the active surveillance management did (20).

6. The Ki-67 LI was higher in PMCs resected due to tumor enlargement than in PMCs without tumor enlargement (26).

7. The total cost of immediate surgery, including the costs for salvage surgery and postoperative care for 10 years, was 4.1 times the total cost of 10-year management for active surveillance (31).

TSH, thyrotropin.

disease-free survival, but only old age was an independent predictor of carcinoma death in patients with clinical PTCs (17). Together, these findings indicate that older PTC patients have significantly poorer prognoses than young patients.

In 2013, Miyauchi et al. demonstrated that the percentages of biochemically persistent disease of PTC patients who underwent total thyroidectomy were significantly higher in patients $<40$ years and patients $\geq 60$ years compared to those aged $40-60$ years. The incidence of patients with a $\mathrm{Tg}$ doubling time (TgDT) $<2$ years, which indicates rapid tumor growth, increased with age (18). The cutoff ages of 40 and 60 years were therefore considered as appropriate to evaluate the biological characteristics of PTCs. In a 2014 study, patient ages were thus divided into three categories - young ( $<40$ years), middle ( $40-59$ years), and old age ( $\geq 60$ years) — in order to investigate the relationship between age and PMC progression (15). In contrast to clinical PTC, the incidences of size enlargement and the novel appearance of node metastasis were significantly higher in young patients compared to middle-aged and old patients, and these incidences decreased with age at presentation. These findings were demonstrated by both univariate and multivariate analyses. Sex, family history, and multiplicity were not found to be predictors of PMC progression in the multivariate analysis. It is very interesting that although clinical PTCs in old patients are often progressive and should be treated carefully, low-risk PMCs in old patients are the best candidates for active surveillance.

In 2010, Sugitani et al. at the CIH reported that only $7 \%$ of 300 low-risk PMCs in a cohort of 230 patients enlarged during active surveillance (19). They also noted that only $1 \%$ of their 230 patients showed novel lymph node metastases during active surveillance. Their series included a higher percentage of old patients than the series at Kuma Hospital (15), since their patients were seen at a cancer center. However, the studies from the two institutions clearly show that during active surveillance, the incidences of tumor-size increase and the novel appearance of nodal metastasis in low-risk PMCs are low, and none of the active-surveillance patients showed distant metastasis or died of thyroid carcinoma. Both studies also showed that rescue surgery after progression signs were detected was not too late and that no life-threatening recurrence was detected thereafter. As rescue surgery for tumor enlargement, a hemithyroidectomy with paratracheal dissection was performed if the tumor was solitary. For cases with the appearance of nodal metastasis, a total thyroidectomy with dissection of the involved lateral neck compartment as well as the central neck compartment was performed. These are important points for adopting an active-surveillance policy as a low-risk PMC management option. Table 2 summarizes the two institutions' important findings regarding active surveillance.

\section{Appearance of novel lymph node metastasis during active surveillance}

In the active surveillance of 1235 low-risk PMC patients, $3.8 \%$ of patients showed novel lymph node metastasis at 10 years of active surveillance. One might think that the appearance of nodal metastasis is a failure of active surveillance. If these patients had been treated at their presentation, the most likely procedure would have been hemithyroidectomy with/ without paratracheal dissection. This procedure would be unlikely to prevent the appearance of nodal metastasis in the lateral neck compartments. However, this did actually occur in the immediate-surgery series, as described later $(15,20)$. These patients required a second operation: completion thyroidectomy with modified neck dissection. It is thought that one operation (i.e., total thyroidectomy with modified neck 


\section{Obtuse angle High-risk}

FIG. 4. Schema of typical examples of PMCs presenting a high, intermediate, and low risk for trachea invasion.

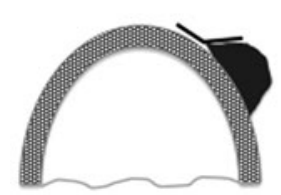

Nearly right angle or unclear Intermediate risk

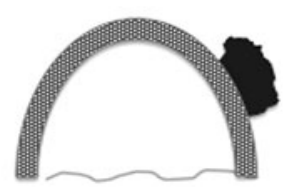

Acute angle Low-risk

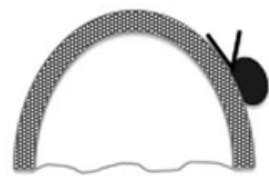

dissection) is better than two operations, since their final outcomes would be similarly excellent (21).

\section{Active Surveillance: Clinical Topics}

\section{Evaluating the possibility of tracheal or RLN invasion}

As described above, the location of PMCs is one of the most important issues in deciding whether active surveillance is appropriate. Tumors presenting tracheal invasion and/or RLN invasion should be operated on immediately. To evaluate the possibility of tracheal or RLN invasion, a CT scan is often more useful than ultrasound. For tumors located in the thyroid lobe's dorsal part, a CT scan may help during the evaluation.

The data suggest that the most important findings for evaluating the possibility of tracheal invasion are the angle formed by the tumor surface and the tracheal cartilage (Fig. 4) and the tumor size (22). In patients who underwent surgery, none of the PMCs $<7 \mathrm{~mm}$ invaded the trachea. In the PMCs $\geq 7 \mathrm{~mm}, 12 /$ 51 (24\%) PMCs showing obtuse angles with the tracheal cartilage required resection of part of the trachea because of invasion. Although 13/78 (17\%) PMCs showed a nearly right or unclear angle, and 5/208 (2\%) PMCs showed an acute angle requiring resection of peritracheal connective tissue, none of these patients required partial resection of the trachea. Thus, think that if the angle is acute, the patient could be a candidate for active surveillance, whereas an obtuse angle indicates that the patient should be surgically treated because of the suspicion for tracheal invasion.

Whether a tumor invades the RLN is judged based on whether the normal rim of the thyroid is present between the tumor and the RLN's course on ultrasound and a CT scan if necessary (22). In this study, similar to tracheal invasion, PMCs $<7 \mathrm{~mm}$ did not invade the RLN, regardless of the risk classification. Of the PMCs $\geq 7 \mathrm{~mm}, 9 / 98$ (9\%) and 23/98 (23\%) high-risk cases lacking the normal rim required resection of part of the RLN and sharp dissection to preserve the nerve, respectively, whereas none and only 2/28 (7\%) low-risk cases with a normal rim required these procedures, respectively. Of course none of the 748 patients whose PMCs were not located on the RLN's course required these procedures (22).

\section{Ultrasonographic findings of primary tumors}

Fukuoka et al. showed that tumors with an initially rich vascularity had a significantly higher rate of tumor enlargement, but the vascularity of most of the tumors decreased during active surveillance (23). Their multivariate analysis showed that macroscopic or rim calcification and poor vascularity at the initial examination were significantly correlated with non-progressive disease. In a study using surgical specimens, PMCs with an ill-defined edge on ultrasonographic examination were likely to show recurrence (24). However, since no life-threatening recurrence was detected after a rescue surgery, these ultrasound findings do not prevent the inclusion of these patients in an active-surveillance program.

\section{TSH suppression}

Regarding TSH suppression, the data from Kuma Hospital and CIH have been discrepant. Sugitani et al. reported that the serum TSH value does not influence the progression of PMC (25). At Kuma Hospital, physicians have occasionally performed mild TSH suppression (setting the serum TSH value at low normal or slightly lower than the lower normal limit) with levothyroxine administration, especially for young patients, at the physician's discretion. Although the number of patients who underwent TSH suppression is small, none who underwent TSH suppression showed a novel appearance of node metastasis (15). Although the evidence level is not high, mild TSH suppression may be useful to prevent PMC progression, especially for young patients, who carry a higher possibility of disease progression than older patients. It is also noted that most of the patients studied by Sugitani et al. were old and thus had a lower possibility of disease progression. This might be why Sugitani et al. did not observe a significant difference among their study groups.

\section{Lack of PMC progression markers}

Active surveillance of PMCs was initiated partly because there were no markers that can be used to predict at presentation whether a PMC will progress. Active surveillance was the only method to discriminate PMCs that are progressing. This scenario has not changed. Hirokawa et al. reported that the Ki-67 labeling index was higher in PMCs surgically removed after size enlargement during active surveillance compared to PMCs without size enlargement on histopathological examinations, but this was not evaluated in preoperative FNAB specimens (26). The combination of $B R A F$ mutation and TERT promoter mutations in clinical PTC was described as associated with poor prognosis (27). However, these mutations were not investigated using PMC FNAB specimens.

\section{PMC progression and pregnancy}

Low-risk PMCs are sometimes identified in young women who desire children. Shindo et al. reported that the PMCs of four of nine patients showed size enlargement during pregnancy (28). Although that study was reported from Kuma Hospital, it was found that there was a large selection bias in the patient series. The records of all female patients aged $\leq 50$ years who underwent active surveillance were rechecked, and it was found that only $8 \%$ (4/51) of the low-risk PMC 
patients showed size enlargement of $\geq 3 \mathrm{~mm}$ based on measurements performed before pregnancy and after delivery. None of the 51 patients showed a novel appearance of lymph node metastasis. Of the four patients whose PMCs showed size enlargement, only two underwent surgery after delivery, whereas the remaining two continued active surveillance because their tumors showed no further progression thereafter (29). Thus, young females with low-risk PMCs and the possibility of future pregnancies do not need to be excluded from active surveillance. If their tumors enlarge, rescue surgery after delivery would not be too late.

\section{Active Surveillance as the First-Line Management for Low-Risk PMCs}

The question of whether immediate surgery or active surveillance is better for managing low-risk PMC patients is important. The outcomes of these management approaches were compared (20). Among 2153 patients with low-risk PMCs seen at Kuma Hospital, 1179 (55\%) patients chose active surveillance and 974 (45\%) patients underwent immediate surgery. Post surgery, five patients had local recurrence that was successfully treated by salvage surgery. The remaining patients were alive without evidence of disease, but another five patients died of other causes. Of the active-surveillance patients, 94 underwent conversion thyroid surgery for various reasons, including the patient's change of mind. Although one of these patients had a local recurrence requiring salvage surgery, the 94 patients were free of disease. During surveillance, three patients died of other causes, and the remaining 1082 patients were alive without disease progression. No patients developed uncontrollable disease or died of the disease. Thus, the outcomes of these management modalities were similarly excellent.

However, the incidences of unfavorable events (e.g., temporary vocal cord paralysis, permanent or temporary hypoparathyroidism, need for levothyroxine substitution, and a surgical neck scar) were significantly higher in the immediate-surgery patients compared to those who chose active surveillance at their diagnosis. Very unfortunately, two $(0.2 \%)$ of the immediate-surgery patients acquired permanent vocal cord paralysis, although their surgeries were performed by highly experienced endocrine surgeons at Kuma Hospital, a center for thyroid diseases (20). One might think that surgery for low-risk PMC would be easy, and it is indeed simple. However, human error can occur during simple and easy surgeries. It can be speculated that if non-experts had performed these patients' surgeries, the incidence of adverse events would have been much higher. This also contributes to the recommendation for active surveillance as the first-line management for low-risk PMC.

\section{Medical Costs}

Medical costs are a very important societal and individual concern. Lubitz et al. warned that based on the current trend in the incidence of well-differentiated thyroid cancer, the 2013 figure of US\$1.6 billion as the estimated overall societal cost of well-differentiated thyroid cancer care in the United States might exceed US $\$ 3.5$ billion by 2030 (30). The costs of surgery and active surveillance were analyzed based on the data of patients who underwent these management approaches. The total cost of immediate surgery, including the costs for salvage surgery and postoperative care for 10 years, was 4.1 times the total cost of 10-year management with active surveillance, including the costs for conversion surgery and salvage surgery (US\$8437 vs. US\$2052 per person) (31).

Costs such as those for physician visits, examinations, surgeries, and prescription medications vary greatly among countries. Insurance systems also vary greatly among countries and even within a given country. However, in Japan, the vast majority of clinical practices are conducted under the Japanese Health Care Insurance system, in which the cost for any aspect of clinical practice is the same, regardless of the experience of the physician or the size and location of the hospital. Therefore, the results of this cost analysis apply universally within Japan. If one wants to calculate the costs incurred in a given healthcare facility, the flow model and cost table provided in a previous study can be used (31).

\section{Discussion}

The prevalence of ultrasound and ultrasound-guided FNAB has greatly aided the detection and diagnosis of small nodules, including low-risk PMCs. However, ultrasound screening often detects many low-risk PMCs, which tend to be harmless. In 1997, Miyauchi contended that thyroidscreening studies using ultrasound would result in more harm than good. He proposed: (i) not using ultrasound for thyroid screening studies; (ii) that even if ultrasound is used, the threshold at which thyroid nodules should be described, even if seen on the ultrasound, should be set at a larger size; (iii) even if nodules are described, the indications for FNAB should be limited to larger and suspicious nodules; and (iv) if PMCs are diagnosed, active surveillance for low-risk PMCs should be considered (32). He also tentatively recommended that nodules $\leq 1 \mathrm{~cm}$ should not be evaluated with FNAB unless they have other malignant signs such as lymph nodes and/or distant metastasis or if the patient show symptoms such as RLN paralysis. These suggestions are similar to those in the 2015 ATA guidelines published 18 years later (11).

Several conditions are required for implementing active surveillance for low-risk PMCs. In order to implement this management, the Memorial Sloan Kettering Cancer Center group proposed a risk stratification of PMCs according to a clinical framework in collaboration with the authors (33). They divided patients into three categories: ideal, appropriate, and inappropriate candidates according to age, sex, tumor multiplicity, location of tumor, and signs of extrathyroidal extension or metastasis. This risk stratification might help physicians and patients to choose a course of management for PMC. Of course, the fully informed decision by the patient in selecting the management modality should be highly respected. However, patients' decisions are strongly influenced by how they are informed and who informs them. Clinicians should be confident about the active surveillance and immediate surgery outcomes described above. With the proper information from physicians, patients can be expected to make the decision most appropriate for them.

Educating physicians, surgeons, patients, and the public about how active surveillance of low-risk PMCs can be an excellent management modality is important. In addition, the availability of skilled sonographers is essential. Accurate ultrasonographic findings obtained by a skilled sonographer are necessary for evaluating low-risk PMC. A system for the follow-up of patients at outpatient clinics is also mandatory. 
A recall system by mail or another form of contact is necessary for patients who do not comply with regular checkups.

Lastly, a simple tip for helping ensure that patients with small thyroid nodules thoroughly understand their condition and options is that the necessary information should be provided to the patient in a brochure about PMCs and treatment options before the FNAB is performed.

One may argue that active surveillance only delays surgical intervention. Based on the age decade-specific disease progression rate at the 10-year active surveillance point, calculated using Kaplan-Meier curves, the lifetime probability of disease progression (defined as tumor enlargement of $\geq 3 \mathrm{~mm}$ and/or the appearance of node metastasis) was estimated. The estimated values were $48.9 \%$ for patients in their 20 s at presentation, $25.7 \%$ for those in their 30 s, $21.4 \%$ in their $40 \mathrm{~s}, 11.4 \%$ in their $50 \mathrm{~s}, 8.3 \%$ in their $60 \mathrm{~s}$, and $3.5 \%$ for those in their 70s (Miyauchi A, Kudo T, Ito Y, et al. 2016 Estimation of the lifetime probability of disease progression of papillary microcarcinoma of the thyroid during active surveillance. Surgery; accepted). One might think that the values $48.9 \%$ and $25.7 \%$ in the 20 s and 30 s are too high to accept active surveillance. However, these estimates indicate that $>50 \%$ and approximately $75 \%$ of the patients in these age decades would not require surgical intervention during their lifetimes, and the vast majority of the patients in their 40s or older would not require surgical intervention during their lifetimes. The benefits of delayed surgical intervention are also that patients can undergo surgery when their life circumstances come to be better and patients can keep normal thyroid function for many more years.

There are some therapeutic options other than active surveillance and immediate surgery such as percutaneous ethanol injection therapy (PEIT) and radiofrequency ablation (RFA) $(34,35)$. These therapy options are adopted in order to control primary lesions. However, it was demonstrated that up to $40 \%$ of patients with PMC have pathological nodal metastases in the central compartment or even in the lateral compartment if these compartments are dissected (13). These nodal metastases cannot be treated with PEIT or RFA. Treating only primary lesions by PEIT or RFA might result in losing a biomarker of the disease. In the authors' opinion, active surveillance is more appropriate for triaging PMC that may have aggressive behavior, and a rescue surgical treatment should be done as the second line of therapy for PMCs that may grow.

Based on the accumulation of data, it can now be said that active surveillance of low-risk PMCs is a safe procedure and much more beneficial than immediate surgery for patients and for society. The data support that active surveillance of low-risk PMCs should be the first-line management modality because only a small percentage of low-risk PMCs progress, and rescue surgery after progression signs are detected has not caused significant recurrence of these carcinomas.

\section{Acknowledgments}

The late Dr. Kanji Kuma (1927-2008), the former President of Kuma Hospital, eagerly supported the active-surveillance clinical trial. Without his agreement and permission, the present studies would not have been possible.

\section{Author Disclosure Statement}

No competing financial interests exist.

\section{References}

1. Davies L, Welch HG 2006 Increasing incidence of thyroid cancer in the United States, 1973-2002. JAMA 595: 2164-2167.

2. Davies L, Welch HG 2014 Current thyroid cancer trends in the United States. JAMA Otolaryngol Head Neck Surg 140: 317-322.

3. Ahn HS, Kim HJ, Welch HG 2014 Korea's thyroid-cancer "epidemic" - screening and overdiagnosis. N Engl J Med 371:1765-1767.

4. Takami H, Ito Y, Okamoto T, Yoshida A 2011 Therapeutic strategy for differentiated thyroid carcinoma in Japan based on a newly established guideline managed by Japanese Society of Thyroid Surgeons and Japanese Association of Endocrine Surgeons. World J Surg 35:111-121.

5. Sugitani I, Fujimoto Y 1999 Symptomatic versus asymptomatic papillary thyroid microcarcinoma: a retrospective analysis of surgical outcome and prognostic factors. Endocr J 46:209-216.

6. Ito $\mathrm{Y}$, Masuoka $\mathrm{H}$, Fukushima $\mathrm{M}$, Inoue $\mathrm{H}$, Kihara $\mathrm{M}$, Tomoda C, Higashiyama T, Takamura Y, Kobayashi K, Miya A, Miyauchi A 2010 Excellent prognosis of patients with solitary T1N0M0 papillary thyroid carcinoma who underwent thyroidectomy and elective lymph node dissection without radioiodine therapy. World J Surg 34: 1285-1290.

7. Ito Y, Miyauchi A 2007 A therapeutic strategy for incidentally detected papillary microcarcinoma of the thyroid. Nat Clin Pract Endocrin Metab 3:240-248.

8. Takebe K, Date M, Yamamoto Y 1994 Mass screening for thyroid cancer with ultrasonography [in Japanese]. KARKINOS 7:309-317.

9. Vaccarella S, Dal Maso L, Laversanne M, Bray F, Plummer M, Franceschi S 2015 The impact of diagnostic changes on the rise in thyroid cancer incidence: a population-based study in selected high-resource countries. Thyroid 25:1127-1136.

10. Lim H, Devesa SS, Sosa JA, Check D, Kitahara CM 2017 Trends in thyroid cancer incidence and mortality in the United States, 1974-2013. JAMA 317:1338-1348.

11. Haugen BR, Alexander EK, Bible KC, Doherty GM, Mandel SJ, Nikiforov YE, Pacini F, Randolph GW, Sawka AM, Schlumberger M, Schuff KG, Sherman SI, Sosa JA, Steward DL, Tuttle RM, Wartofsky L 20162015 American Thyroid Association management guidelines for adult patients with thyroid nodules and differentiated thyroid cancer. Thyroid 26:1-133.

12. Uruno T, Miyauchi A, Shimizu K, Tomoda C, Takamura Y, Ito Y, Miya A, Kobayashi K, Matuzuka F, Amino N, Kuma K 2005 Usefulness of thyroglobulin measurement in fineneedle aspiration biopsy specimens for diagnosing cervical lymph node metastasis in patients with papillary thyroid cancer. World J Surg 29:483-485.

13. Ito $\mathrm{Y}$, Uruno R, Nakano K, Takamura Y, Miya A, Kobayashi K, Yokozawa T, Matsuzuka F, Kuma S, Kuma K, Miyauchi A 2003 An observation trial without surgical treatment in patients with papillary microcarcinoma of the thyroid. Thyroid 13:381-388.

14. Ito $\mathrm{Y}$, Miyauchi A, Inoue $\mathrm{H}$, Fukushima M, Kihara M, Higashiyama T, Tomoda C, Takamura Y, Kobayashi K, Miya A 2010 An observation trial for papillary thyroid microcarcinoma in Japanese patients. World J Surg 34: 28-35.

15. Ito Y, Miyauchi A, Kihara M, Higashiyama T, Kobayashi K, Miya A 2014 Patients age is significantly related to the 
progression of papillary microcarcinoma of the thyroid under observation. Thyroid 24:27-34.

16. Ito Y, Kudo T, Kobayashi K, Miya A, Ichihara K, Miyauchi A 2012 Prognostic factors for recurrence of papillary thyroid carcinoma in the lymph nodes, lung, and bone: analysis of 5,768 patients with average 10-year follow-up. World J Surg 36:1274-1278.

17. Ito Y, Miyauchi A, Tomoda C, Hirokawa M, Kobayashi K, Miya A 2014 Prognostic significance of young age in papillary thyroid carcinoma: analysis of 5,733 patients with 150 months' follow-up. Endocr J 61:491-497.

18. Miyauchi A, Kudo T, Kihara M, Higashiyama T, Ito Y, Kobayashi K, Miya A 2013 Relationship of biochemically persistent disease and thyroglobulin-doubling time to age at surgery in patients with papillary thyroid carcinoma. Endocr J 60:415-421.

19. Sugitani I, Toda K, Yamada K, Yamamoto N, Ikenaga M, Fujimoto Y 2010 Three distinctly different kinds of papillary thyroid microcarcinoma should be recognized: our treatment strategies and outcomes. World J Surg 34: 1222-1231.

20. Oda H, Miyauchi A, Ito Y, Yoshioka K, Nakayama A, Sasai H, Masuoka H, Yabuta T, Fukushima M, Higashiyama T, Kihara M, Kobayashi K, Miya A 2016 Incidences of unfavorable events in the management of low-risk papillary microcarcinoma of the thyroid by active surveillance versus immediate surgery. Thyroid 26:150-155.

21. Miyauchi A 2016 Clinical trials of active surveillance of papillary microcarcinoma of the thyroid. World J Surg 40: 516-522.

22. Ito Y, Miyauchi A, Oda H, Kobayashi K, Kihara M, Miya A 2016 Revisiting low-risk thyroid papillary microcarcinomas resected without observation: was immediate surgery necessary? World J Surg 40:523-528.

23. Fukuoka O, Sugitani I, Ebina A, Toda K, Kawabata K, Yamada K 2016 Natural history of asymptomatic papillary thyroid microcarcinoma: time-dependent changes in calcification and vascularity during active surveillance. World $\mathrm{J}$ Surg 40:529-537.

24. Ito Y, Kobayashi K, Tomoda C, Uruno T, Takamura Y, Miya A, Matsuzuka F, Kuma K, Miyauchi A 2005 Illdefined edge on ultrasonographic examination can be a marker of aggressive characteristic of papillary thyroid microcarcinoma. World J Surg 29:1007-1011.

25. Sugitani I, Fujimoto Y, Yamada K 2014 Association between serum thyrotropin concentration and growth of asymptomatic papillary thyroid microcarcinoma. World J Surg 38:673-678.

26. Hirokawa M, Kudo T, Ota H, Suzuki A, Miyauchi A 2016 Pathological characteristics of low-risk papillary thyroid microcarcinoma with progression during active surveillance. Endocr J 63:805-810.

27. Xing M, Liu R, Liu X, Murugan AK, Zhu G, Zeiger MA, Pai S, Bishop J 2014 BRAF V600E and TERT promoter mutations cooperatively identify the most aggressive papillary thyroid cancer with highest recurrence. J Clin Oncol 32:2718-2725.

28. Shindo H, Amino N, Ito Y, Kihara M, Kobayashi K, Miya A, Hirokawa M, Miyauchi A 2014 Papillary thyroid microcarcinoma might progress during pregnancy. Thyroid 24:840-844.

29. Ito Y, Miyauchi A, Kudo T, Ota H, Yoshioka K, Oda H, Sasai H, Nakayama A, Yabuta T, Masuoka H, Fukushima M, Higashiyama T, Kihara M, Kobayashi K, Miya A 2016 Effects of pregnancy on papillary microcarcinoma of the thyroid re-evaluated in the entire patient series at Kuma Hospital. Thyroid 26:156-160.

30. Lubitz CC, Kong CY, McMahon PM, Daniels GH, Chen Y, Economopoulos KP, Gazelle GS, Weinstein MC 2014 Annual financial impact of well-differentiated thyroid cancer care in the United States. Cancer 120:1345-1352.

31. Oda H, Miyauchi A, Ito Y, Sasai H, Masuoka H, Yabuta T, Fukushima M, Higashiyama T, Kihara M, Kobayashi K, Miya A 2017 Comparison of the costs of active surveillance and immediate surgery in the management of lowrisk papillary microcarcinoma of the thyroid. Endocr $\mathrm{J}$ 64:59-64.

32. Miyauchi A 1997 Mass screening for the thyroid (in Japanese). Jpn J Clin Exp Med 74:1745-1748.

33. Brito JP, Ito Y, Miyauchi A, Tuttle RM 2016 A clinical framework to facilitate risk stratification when considering an active surveillance alternative to immediate biopsy and surgery in papillary microcarcinoma. Thyroid 26:144-149.

34. Lim CY, Yun JS, Lee J, Nam KH, Chung WY, Park CS 2007 Percutaneous ethanol injection therapy for locally recurrent papillary thyroid carcinoma. Thyroid 17:347-350.

35. Dupuy DE, Monchik JM, Decrea C, Pisharodi L 2001 Radiofrequency ablation of regional recurrence from welldifferentiated thyroid malignancy. Surgery 130:971-977.

Address correspondence to: Akira Miyauchi, MD

Department of Surgery Kuma Hospital

8-2-35 Shimoyamate-dori

Chuo-Ku, Kobe

Hyogo 650-0011

Japan

E-mail: miyauchi@kuma-h.or.jp 